\title{
artigo
}

Dutra Filho, W.A.; Paiva, C.M.M.; Delgado, V.H.F.; Lins, U.M.B.L.; França, E.S.;

Uso de INIBIDOR de JAK2 combinado com AINE como uma proposta de tratamento para COVID-19: Uma revisão de literatura

\section{Uso de INIBIDOR de JAK2 combinado com AINE como uma proposta de tratamento para COVID-19: Uma revisão de literatura}

\author{
JAK2 INHIBITORAND NSAIDs combinated use as a treatment proposal for COVID-19: A literature review \\ Uso de INIBIDOR de JAK2 combinado con AINE como propuesta de tratamiento para a COVID-19: Una revisión de la literatura
}

\begin{abstract}
RESUMO
A COVID-19 induz a um processo inflamatório e de eventos trombóticos que geram danos ao organismo. A busca por novos protocolos medicamentosos tem sido severamente pesquisado como meta de combate à doença.Objetivo: Propor, por meio de uma revisão de literatura, o uso combinado do baricitinib e da aspirina para pacientes com doença moderada a severa da COVID-19. Métodos: Foi realizada uma revisão integrativa da literatura nas plataformas Google Search e Pub Med, analisando estudos observacionais e os clínicos de intervenção. Resultados: A média da mortalidade entre os estudos da aspirina foi de 13,25\%, foi observado menor necessidade de ventilação mecânica e menor taxa de admissão em UTI. Já com o baricitinb, a mortalidade foi de 6,25\%, houve diminuição na concentração plasmáticas de IL-6, CRP e de neutrófilos e aumento do número de linfócitos. Conclusão: A associação dos dois medicamentos mostra atividade antiviral, antiinflamatória e antitrombótica, atuando sobre o JAK2 e NFkb simultaneamente.
\end{abstract}

DESCRITORES: COVID-19; Aspirina;Baricitinib;Tratamento.

\section{ABSTRACT}

The search for drugs protocols to the COVID-19 treatment had been severely studied, considering the inflammation states and the thrombotic events. Objective: Propose, through a literature review, the combined use of baricitinib and apirin for patients with moderate to severe COVID-19 disease. .METHODS: An integrative literature review was carried out on Google Search and Pub Med, analyzing observational and clinical intervention studies Results: The average mortality between the studies with the aspirin was $13.25 \%$, it was observed a lower necessity for mechanical ventilation and rate of admission to the ICU. While the baricitinb studies had an average of $6.25 \%$, decrease in plasma concentrations of IL-6, CRP and neutrophils and an increase in the number of lymphocytes. Conclusion: The association of these two drugs shows antiviral, anti-inflammatory and antithrombotic activity, acting on JAK2 and NF-kb simultaneously.

DESCRIPTORS: COVID-19; Aspirin;Baricitinib; Treatment.

\section{RESUMEN}

La búsqueda de protocolos de fármacos para el tratamiento del COVID-19 había sido estudiada de forma rigurosa, considerando tu estado inflamatorio y los eventos trombóticos.ObjetivoProponer, a través de una revisión de la literatura, el uso combinado de baricitinib y aspirina en pacientes conenfermedad COVID-19 de moderada a grave.Método: Se realizó una revisión integradora de la literatura enlas plataformas Google Search y Pub Med, analizando estúdios observacionales y de intervención clínica. Resultados: La mortalidad media entre los dos estúdios del aspirina fuedel 13,25\%, fueobservado menor necesidad de ventilaciónmecánica y menor tasa de ingresoen UCI. Mientras, os estúdios conelbaricitinibtienes uma media de 6,25\%, disminución em lasconcentraciones plasmáticas de IL-6, PCR, número de neutrófilos y un aumento enel número de linfocitos. Conclusión: La asociación de losmedicamientosmuestraactividad antiviral, antiinflamatoria y antitrombótica, actuando sobre JAK2 y NF-kbsimultáneamente.

DESCRIPTORES: COVID-19; Aspirina;Baricitinib; Tratamiento.

RECEBIDO EM: 16/12/2020 APROVADO EM: 19/01/2021

\section{Williams Alexandre Dutra Filho}

Graduando de Odontologia, UNINASSAU - Recife.

ORCID: 0000-0002-6865-6685 


\section{Camila Maria Miranda de Paiva}

Graduanda de Odontologia, UNINASSAU - Recife.

ORCID: 0000-0001-9744-764X

\section{Vitória Helen Feliciano Delgado}

Estudante de Odontologia da UNINASSAU- Recife (PE).

ORCID: 0000-0002-2829-1685

\section{Uiara Maria de Barros Lira Lins}

Graduada em Ciências Farmacêuticas, Mestranda em Desenvolvimento de Processos Ambientais pela Universidade Católica de Pernambuco (UNICAP).

ORCID: 0000-0002-6007-9932

\section{Eduardo da Silva França}

Mestrando em Desenvolvimento de Processos Ambientais pela Universidade Católica de Pernambuco. ORCID: 0000-0003-1573-5133

\section{INTRODUÇÃO}

0 vírus SARS CoV-2 é o agente causador da doença COVID-19. Ele é responsável por provocar doenças respiratórias graves. Contudo, também é capaz de atingir vários outros sistemas. Já sendo reportado maior suscetibilidade por eventos trombóticos em pacientes com a doença severa, sobretudo em pequenos vasos sanguíneos. A evolução clínica da doença pode ser associada à desregulação imune do indivíduo, podendo sair de uma resposta de proteção ao organismo para uma incontrolada produção de mediadores e células da inflamação. A inflamação causada pelo vírus é a principal causa de mortalidade nas pessoas acometidas pela doença. ${ }^{1,2}$

Devido a esses eventos inflamatórios, vários medicamentos como antiinflamatórios e antivirais, têm sido testados na COVID $-19^{3}$ dentre eles os inibidores da rota de sinalização JAK-STAT têm destaque pela sua atividade notável de controle da produção das citocinas envolvida no processo da inflamaçãó ${ }^{4}$. Já o Ácido Acetil Salicílico (AAS) classificado como Antiinflamatório Não Esteróide (AINE) tem sido considerado promissor por atuar sobre os mediadores da inflamação e prevenir eventos trombóticos. ${ }^{5}$

Considerando a atividade antiviral e antiinflamatória do baricitinib e do AAS bem como os resultados promis- sores no tratamento da COVID-196, foi realizadaumarevisão integrativa da literatura para buscar dados sobre a eficácia de cada tratamento individualmente. Propondo a realização de um possível estudo clínico utilizando esses dois medicamentos associados para o controle da inflamação, da carga viral e da trombose causada em pacientes severos para COVID-19.

\section{MÉTODOS}

Foi realizadauma revisão do tipo integrativa da literatura para identificar a produção científica relacionada com a utilização do AAS e do baricitinib para o tratamento COVID-19. O levantamento bibliográfico foi realizado nas bases de dados: PubMed e Google Search, realizado no período de setembro a outubro de 2020. O idioma utilizado para busca foi a língua inglesa. Foram utilizados descritores presentes nos Descritores em Ciências da Saúde (DeSC). Assim, para os trabalhos sobre o uso da AAS na COVID-19 foram utilizados os descritores "Aspirin"; “COVID-19”. Já os relacionados com a terapêutica do baricitinib na doença, foram utilizados os descritores "Baricitinib"; "COVID-19". Foram incluídos os artigos de estudos observacionais (caso-controle,transversais e de corte), além estudos de intervenção (ensaios clínicos controlados, não controlados e ensaio cego), utilizando esses dois medicamentos em pacientes positivos para a COVID-19. Os artigos excluídos para a análise foram os de revisão de literatura (revisão sistemática, integrativa, meta análise) sobre a aplicabilidade desses medicamentos para o tratamento da doença.

Foram coletados dos estudos dados sobre o número de pessoas investigadas em cada trabalho, o estado da doença que elas apresentavam no momento da admissão no hospital (leve, médio, moderado e severo), taxa de mortalidade dos pacientes após o uso desses medicamentos, melhoras dos sintomas e sobre dados do hemograma sobre o aumento/redução de mediadores e células inflamatórias após. Ao final os resultados dos estudos foram comparados entre si e foi realizada uma análise do comportamento desses parâmetros citados acimas ao final do tratamento com os medicamentos.

\section{RESULTADOS}

O número total de estudos encontrados nas plataformas foi de 136 trabalhos (78 do baricitinib e $58 \mathrm{da}$ aspirina), sendo que destes 6 foram selecionados, nos quais 4 eram estudos clínicos usando o baricitinib e 2, a aspirina.

O total de pacientes estudados nos trabalhos analisados com o uso do Ácido Acetil Salicílico (AAS) somaram 133. A 


\section{artigo}

Dutra Filho, W.A.; Paiva, C.M.M.; Delgado, V.H.F.; Lins, U.M.B.L.; França, E.S.;

Uso de INIBIDOR de JAK2 combinado com AINE como uma proposta de tratamento para COVID-19: Uma revisão de literatura

maior parte teve a doença de média a severa com um taxa de mortalidade média entre os estudos de $13,25 \%$. Como mostrado na tabela 1 .

No estudo de Florêncio e colaboradores (2020) atorvastatin ou com o rusorvastatin. Todos os pacientes tiveram uma remissão significativa dos sintomas, e se tornaram sintomático ou oligosintomático e, não houve eventos hemorrágicos com a aplicação desse medicamento. A comparação entre o uso e não uso da aspirina como tratamento para a COVID-19 foi descrito por CHOW e colaboradores (2020), houve uma menor precisão de ventilação mecânica, admissão na UTI para o primeiro grupo.

O total de pacientes alisados foram 180, distribuídos entre os casos, moderado e severo. Com taxa de mortalidade média entre os estudos de 6,25\%. Nos 4 estudos, os pacientes foram tratados de 2 à $4 \mathrm{mg}$ de baricitnib com um tempo de tratamento variando de 1 a 2 semanas (Tabela 2).

No grupo de 15 pessoas de Titanji e colaboradores (2020), 86,7\% dos participantes tiveram uma redução significativa na temperatura corporal nos 5 primeiros dias de terapia, nos níveis de CRP e IL-6. No trabalho do Cantini (2020), foi revelado a diminuição, em 2 semanas de tratamento, de níveis plasmáticos de proteína C- reativa (CRP) em $\mathrm{mg} / \mathrm{ml}$, diminuição do número de neutrófilos em $\mathrm{x} 9 / \mathrm{L}$ e aumento do número de linfócitos em x9/L. Quanto às citocinas, o estudo de Bronte e colaboradores (2020) mostrou uma diminuição de células pró-inflamatórias, IL-1 $\beta$, IL-6 e TNF, dos primeiros 7 dias do tratamento ( $\mathrm{t} 0$ - $\mathrm{t} 7$ ), assim como da CRP. Houve um aumento no número de linfócitos circulantes, de células T CD4+, de células de memória (CD3+CD4+CD45RA-CD27- ), e células B. Já em relação aos ní-

\begin{tabular}{|c|c|c|c|c|}
\hline ESTUDO & INDIVÍDUOS & $\begin{array}{l}\text { ESTADO DA } \\
\text { DOENÇA }\end{array}$ & $\begin{array}{c}\text { DOSE } \\
\text { ADMINISTRADA }\end{array}$ & MORTALIDADE \\
\hline $\begin{array}{l}\text { Florêncio et. al } \\
\text { (2020) }\end{array}$ & 35 & Média & $\begin{array}{l}\text { inicial de } 200 \text { a } 300 \\
\text { mg seguido de doses } \\
\text { diárias de } 100 \text { mg } \\
\text { até o } 15^{\circ} \text { ou } 21^{\circ} \text { da }\end{array}$ & $0 \%$ \\
\hline $\begin{array}{l}\text { CHOW et. al } \\
\text { (2020) }\end{array}$ & 98 & $\begin{array}{c}\text { Moderada a } \\
\text { severa }\end{array}$ & $\begin{array}{c}\text { Dose única (não } \\
\text { especificada) }\end{array}$ & $26,5 \%$ \\
\hline
\end{tabular}

Tabela 2: sobre os estudos com a utilização do baricitinib em pacientes positivos para a COVID-19

\begin{tabular}{|c|c|c|c|c|}
\hline ESTUDO & $\begin{array}{l}\text { NÚMERO } \\
\text { DE } \\
\text { PESSOAS }\end{array}$ & $\begin{array}{l}\text { ESTÁGIO DA } \\
\text { DOENÇA NA } \\
\text { ADMISSÃO }\end{array}$ & $\begin{array}{l}\text { DOSE ADMINISTRADA E } \\
\text { COMP }\end{array}$ & $\begin{array}{l}\text { MORTALIDADE } \\
(\%)\end{array}$ \\
\hline $\begin{array}{l}\text { Bronte et. al } \\
(2020)\end{array}$ & 20 & $\begin{array}{l}\text { Não infor- } \\
\text { mado }\end{array}$ & $\begin{array}{c}\text { Baricinib } 2-4 \text { mg/dia (7 } \\
\text { dias) }\end{array}$ & $5 \%$ \\
\hline $\begin{array}{l}\text { Titanji et. al } \\
\text { (2020) }\end{array}$ & 15 & $\begin{array}{l}\text { Moderado a } \\
\text { severo }\end{array}$ & $\begin{array}{l}\text { Baricitinib } 2-4 \mathrm{mg} / \mathrm{dia} \\
\text { (7 dias) }\end{array}$ & $20 \%$ \\
\hline $\begin{array}{l}\text { Cantini et al. } \\
\text { (2020) }\end{array}$ & 113 & moderado & $\begin{array}{c}\text { Baricitinib } 4 \text { mg/dia } \\
\text { +lopinavir/ritonavir } 250 \\
\text { mg (14 dias) }\end{array}$ & $0 \%$ \\
\hline $\begin{array}{l}\text { Cantini et al. } \\
(2020)\end{array}$ & 12 & moderado & $\begin{array}{l}\text { Baricitinib +lopinavir/rito- } \\
\text { navir- } 250 \text { mg (14 dias) }\end{array}$ & $0 \%$ \\
\hline
\end{tabular}

veis plasmáticos dos antígenos $\operatorname{Ig} \mathrm{A}$ e $\operatorname{IgG}$, houve um aumento do número de IgG.

\section{DISCUSSÃO}

A associação de medicamentos na COVID-19 foi observada nos artigos analisados. Nostrabalhos do Cantini e colaboradores $^{6,7}$ usou o baricitinib com o lopinavir/ritonavir e no grupo controle usou a hidroxicloroquina com esses antivirais, já Titanji e colaboradores8 usaram uma combinação de hidroxicloroquina e baricitinib. Florêncio e colaboradores? usaram o AAS com atorvastatin ou com o rusorvastatin.

O AAS tem como metabólito principal o ácido salicílico, ele é um inibidor não seletivo das cicloxigenases $\mathrm{COX}-1 \mathrm{e}$ COX-2, responsáveis pela produção de mediadores como: prostaglandinas (PG) e tramboxano A2 (TXA2). A acetilação da COX-1 no resíduo de serina 530 ou da COX-2, na ser 516, dimuindo assim a síntese de deprostanoidescomo PGG2 e PGH2 e TXA2. A inibição do TXA2diminui a geração de plaquetas e o infiltrado de neutrófilos no pulmão. Porém, em doses altas, essa efetividade não é mantida, pois há uma interação do AAS com a PGI2, que, por sua vez, desempenha um papel contrário ao TXA2. ${ }^{5,10,11}$

Outros mecanismos seriam: supressão da via de sinalização intracelular NF-kb, inativando a proteína $\mathrm{IKKb}$, que está relacionado com grande parte da produção de citocinas pró-inflamatórias e a consequente recrutação de células da inflamação e com a diferenciação das células $\mathrm{T}$ CD 4+. ${ }^{12,13}$ Além disso, há a acetilação da molécula iNOS, a indução da expressão de da heme oxigenase ( $\mathrm{HO})$, e acetilação de fribrogênio, promovendo a fibrionólise.A atividade antiviral pelo salicicato, o qual pode inibir a replicação viral por bloquear a produção da prostaglandina E2 (PGE2) nos macrófagos e aumenta a regulação dos interferons, também é dada pela inibição da rota intracelular NF-kb. ${ }^{5}$

O baricitinib tem um amplo espectro de inibição de citocinas, ele atua na inibição da rota intracellular JAK2, tendo um 
papel na inibição de citocinas pró-inflamatórias, e mostra atuar no impedimento da endocitose viral do SARS-cov-2 nas células hospedeiras. 14 Ele impede a ativação de células Th 17 pelas citocinas IL-6 e IL-23, os quais levam à produção de proteínas inflamatórias como:, G-CSF, IL-17, , IL-1 $\beta$, IL-6, TNF $\alpha$; quimiocitocinas KC, MIP2A, IL-8, IP10, MIP3A e na ativação das metaloproteinases. ${ }^{15} \mathrm{O}$ baricitinib também atua na inibição da produção da angiotensina II (ANG II), responsável pela vasocontrição, hipertensão e danos crónicos ao pulmão e aumento da infiltração de células da imunidade com a sua produção de citocinas pró-inflamatórias. ${ }^{15,16}$ Todavia, RICHARDSON e colaboradores ${ }^{17}$ defendem em seus estudos que o uso deste medicamento deve ser dado a pacientes com a doença de moderada a severa.
O mecanismo antiviral desse inibidor é resultado da interação química do baricitinib com a proteina AAK1 (do inglês AP2-associated proteinkinase), essa molécula é integrante da família dos NAK, assim como a BIKE, GAK e KTK16, as quais, destacadamente a AAK1 e a GAK estão ligadas aos receptores ACE2, o que permite a endocitose do SARS-CoV-2 por meio da proteína $S$ do vírus. $\mathrm{O}$ baricitinib mostra afinidade contra a proteína $\mathrm{AAK} 1(8,2 \mathrm{nM}), \operatorname{BIKE}(20 \mathrm{nM}) \mathrm{e}$ $\operatorname{GAK}(120 \mathrm{nM}) \cdot{ }^{14,15}$

\section{CONCLUSÃO}

Ensaios clínicos com o baricitinib e o AAS obtiveram resultados promissores em pacientes com doença média a severa. No grupo do AINE as variáveis: menor necessidade de ventilação mecânica, menor taxa de admissão em UTI e não houve eventos hemorrágicos com o uso. O tratamento com o baricitinib mostrou, em linhas gerais, menor concentração plasmáticas de IL-6, CRP, número de neutrófilos e aumento do número de linfócitos, sendo mais efetivo adicionado de lor. Diante dos resultados de cada grupo de medicamento estudado e considerando as abordagens teóricas desenvolvidas por vários autores na discussão sobre o mecanismo de ação dessas substâncias sobre a infecção pelo SARS-CoV-2, foi encontrado um caminho promissor para a associação dos medicamentos por apresentar atividade antiviral, anti-inflamatória e antitrombótica, por atuar conjuntamente na inibição rotas intracelular JAK2 e NF-kb, podendo aumentar o espectro da inibição de citocinas, permitir a não agregação plaquetária e bloquear a entrada e replicação do vírus. -

\section{REFERÊNCIAS}

1. García LF. Immune Response, Inflammation, and the Clinical Spectrum of COVID-19. Front Immunol. 2020;11:1441

2. Song P, Li W, Xie J, Hou Y, You C. Cytokine storm induced by SARS-CoV-2. Clin Chim Acta. 2020; 7: 509:280.

3. Stebbing , Phelan A, Griffin I. COVID-19: combining antiviral and anti-inflammatory treatments.Lancet Infect Dis. 2020; 20: 400-402

4. Seif F, Aazami H, Khoshmirsafa M, Kamali M, Mohsenzadegan M, Pornour M, Mansouri D: JAK Inhibition as a New Treatment Strategy for Patients with COVID-19. Int Arch Allergy Immunol 2020;181:467-475.

5. Bianconi, V., Violi, F., Fallarino, F. et al. Is Acetylsalicylic Acid a Safe and Potentially Useful Choice for Adult Patients with COVID-19 ?. Drugs 2020; 80, 1383-1396.

6. Yang CW., Lee YZ., Hsu HY. Targeting Coronaviral Replication and Cellular JAK2 Mediated Dominant NF- B Activation for Comprehensive and Ultimate Inhibition of Coronaviral Activity. Sci Rep 2017; 7:4105.

7. Cantini F, Niccoli L, Matarrese D, Nicastri E, Stobbione P, Goletti D. Baricitinib therapy in COVID-19: A pilot study on safety and clinical impact. J Infect. 2020 ;81(2):318-356.

8. Cantini F, Niccoli L, Nannini C, et al. Beneficial impact of Baricitinib in COVID-19 moderate pneumonia; multicentre study. J Infect. 2020;81(4):647-679.

9. Titanji BK, Farley MM, Mehta A, Connor-Schuler R, Moanna A, Cribbs SK, O'Shea J, DeSilva K, Chan B, Edwards A, Gavegnano C, Schinazi RF, Marconi VC. Use of Baricitinib in Patients with Moderate and Severe COVID-19. Clin Infect Dis. 2020 Jun; 29: 879.
10. Florencio, Francisco Kleyton Zacarias et al .Aspirin with or without statin in the treatment of endotheliitis, thrombosis, and ischemia in coronavirus disease. Rev. Soc. Bras. Med. Trop., 2020; 53.

11. Chow JH, Khanna AK, Kethireddy S, Yamane D, Levine A, Jackson AM, McCurdy MT, Tabatabai A, Kumar G, Park P, Benjenk I, Menaker J, Aspirin Use is Associated with Decreased Mechanical Ventilation, ICU Admission, and In-Hospital Mortality in Hospitalized Patients with COVID-19. AnesthAnalg. 2020.

12. Liu, T., Zhang, L., Joo, D. et al. NF- B signaling in inflammation. Sig Transduct Target Ther 2017; 2: 17023.

13. Shi C, Zhang N, Feng Y, Cao J, Chen X, Liu B: Aspirin Inhibits IKK- $\beta$-mediated Prostate Cancer Cell Invasion by Targeting Matrix Metalloproteinase- 9 and Urokinase-Type Plasminogen Activator. Cell PhysiolBiochem2017;41:1313-1324.

14. Zhang L, Shi L, Soars SM, Kamps J, Yin H. Discovery of Novel Small-Molecule Inhibitors of NF- B Signaling with Antiinflammatory and Anticancer Properties. J Med Chem, 2018;61(14):5881-99.

15. Peter J Richardson, Mario Corbellino, Justin Stebbing. Baricitinib for COVID-19: a suitabletreatment? 2020; 20: 1013-1014

16. Bronte $V$, Ugel $S$, Tinazzi $E$, Vella $A$, De Sanctis $F$, Canè $S$, Batani $V$, Trovato $R$, Fiore $A$, Baricitinib restrains the immune dysregulation in patients with severe COVID-19. J Clin Invest. $2020 ; 130(12): 6409-6416$.

17. Richardson P, Griffin I, Tucker C, Smith D, Oechsle O, Phelan A, Rawling M, Savory E, Stebbing J. Baricitinib as potential treatment for 2019-nCoV acute respiratory disease. Lancet. 2020;395(10223):30-31. 